\title{
ANÁLISE DOS AGLOMERADOS PRODUTIVOS NOS MUNICÍPIOS DE ANGRA DOS REIS, CAMPOS E PETRÓPOLIS NO ESTADO DO RIO DE JANEIRO
}

\section{RESUMO}

Os aglomerados produtivos são atualmente referência constante nas linhas de pesquisa por se transformar em questão estratégica. Cidades com aglomerados produtivos têm desenvolvimento diferenciado em relação às demais cidades. Este desenvolvimento tem influência direta na localização/instalação e gestão das empresas. Este fator permite a organização obter vantagem competitiva devido à proximidade de seus fornecedores e o contato direto com a população local. Com os fornecedores as empresas aumentam a mobilidade da sua cadeia produtiva e se beneficiam da existência de equipamentos de qualidade na rede. Este estudo tem como propósito analisar, avaliar e classificar os aglomerados produtivos das cidades do Estado do Rio de Janeiro, em particular nos municípios de Angra dos Reis, Campos e Petrópolis, observando como alcançar o desenvolvimento local equilibrado beneficie todos os interessados pelos produtos/serviços da cidade. Os aglomerados produtivos devem necessariamente contribuírem para a melhoria do nível de serviço oferecido ao cliente. A metodologia adotada foi à proposta por Suzigan et al (2003) e Britto e Albuquerque (2002), para avaliar, analisar e classificar os aglomerados, além do levantamento bibliográfico para sustentar o estudo. Os resultados apontam que dependendo da importância da atividade econômica para o município, os aglomerados produtivos contribuem grandiosamente para o desenvolvimento local e aumentam as opções de sortido para o consumidor local.

Palavras-chave: Aglomerados; Benefícios; Estratégia; Competitividade.

\section{ANALYSIS OF PARTICLE PRODUCTION IN THE MUNICIPALITIES OF ANGRA DOS REIS, CAMPOS AND PETROPOLIS IN RIO DE JANEIRO}

\begin{abstract}
The productive clusters are currently constant reference in the research lines turn into strategic issue. Cities with production clusters have differentiated development in relation to other cities. This development has a direct influence on the location / installation and management of companies. This factor allows the organization gain a competitive advantage due to proximity to their suppliers and direct contact with the local population. With supplier companies increase the mobility of its supply chain and benefit from the existence of quality equipment in the network. This study aims to analyze, evaluate and classify the productive clusters of cities in the State of Rio de Janeiro, particularly in the municipalities of Angra dos Reis, Campos and Petropolis, to achieve balanced local development that benefits all stakeholders by product / city services. Productive clusters must necessarily contribute to improving the level of service offered to the customer. The methodology adopted was proposed by the Suzigan et al (2003) and Britto and Albuquerque (2002), to assess, analyze and classify the clusters in addition to bibliographic survey to support the study. The results show that depending on the importance of economic activity for the city, the production clusters greatly contribute to local development and increase the range of options for the local consumer.
\end{abstract}

Keywords: clusters, benefits, strategy, competitiveness. 


\section{LA PRODUCCIÓN DE PARTÍCULAS PARA EL DESARROLLO LOCAL COMO ESTRATEGIA PARA REDUCIR COSTES LOGÍSTICOS}

\section{RESUMEN}

Clusters productivos Actualmente referencia constante en la investigación de líneas se convierten en tema estratégico. Ciudades con racimos de producción se han diferenciado de desarrollo en relación con otras ciudades. Este desarrollo tiene una influencia directa en el lugar / instalación y gestión de empresas. Este factor permite a la organización obtener una ventaja competitiva debido a la proximidad a sus proveedores y el contacto directo con la población local. Con las empresas proveedoras aumentan la movilidad de su cadena de suministro y se benefician de la existencia de equipos de calidad en la red. Este estudio tiene como objetivo analizar, evaluar y clasificar los clusters productivos de las ciudades en el Estado de Río de Janeiro, en particular en los municipios de Angra dos Reis, Campos y Petrópolis, para lograr un desarrollo local equilibrado que beneficie a todas las partes interesadas por producto / servicios de la ciudad. Clusters productivos deben contribuir necesariamente a la mejora del nivel de servicio ofrecido al cliente. La metodología adoptada fue propuesta por el Suzigan et al (2003) y Britto y Albuquerque (2002), para evaluar, analizar y clasificar los grupos, además de estudio bibliográfico para apoyar el estudio. Los resultados muestran que, dependiendo de la importancia de la actividad económica para la ciudad, las zonas de actividades productivas contribuyen en gran medida al desarrollo local y aumentar la gama de opciones para el consumidor local.

Palabras clave: Cúmulos; Beneficios; Estrategia; Competitividad.

Sebastião Lourenço dos Santos João ${ }^{1}$ Gustavo Lopes Olivares ${ }^{2}$

\footnotetext{
1 Graduado em Administração pela Universidade Federal Rural do Rio de Janeiro - UFERSA. Brasil. E-mail: selosantos@live.com.pt

${ }^{2}$ Doutor em Engenharia de Produção pela Pontifícia Universidade Católica do Rio de Janeiro - PUC/RJ. Professor da Universidade Federal Rural do Rio de Janeiro - UFERSA. Brasil. E-mail: olivares.rural@gmail.com
} 


\section{INTRODUÇÃO}

O desenvolvimento local tornou-se um assunto constante nas pesquisas, motivadas pela pretensão dos governos em equilibrar as ofertas econômicas e reduzir as desigualdades sociais. Deste modo, os países apostam no fomento das economias regionais com grupos fortes e unidos para reduzir os problemas empresariais para fomentar o comércio e estimular a economia local. Assim, busca-se por mecanismos e estratégias que permitam estimular o crescimento equilibrado das regiões. Observa-se, a procura por vantagens competitivas, como: a diminuição dos custos de produção e de transação; domínio e expansão de mercados e, principalmente, a inovação constante em processos e produtos, possibilitando que as empresas organizadas em torno de aglomerados incorporassem essas vantagens.

Para Amato Neto (2000) a partir dos anos 70 , verificou-se uma clara mudança na organização industrial, no sentido do fortalecimento das relações entre as empresas que formam as aglomerações produtivas. Isso pôde ser comprovado com as experiências de sucesso dos distritos industriais da chamada Terceira Itália, nos sistemas produtivos locais da França, na Alemanha e no Reino Unido, no Vale do Silício nos EUA ou as redes de empresa no Japão, na Coréia e em Taiwan. Cassiolato e Szapiro (2003) afirmam que o conceito de aglomerado de empresas torna-se explicitamente associado à competitividade, principalmente a partir do início dos anos 90, o que parcialmente explica seu forte apelo para os formuladores de políticas públicas e privadas por ser um fenômeno com relação direta ao local onde está inserido.

Existem reconhecidas dificuldades na conceituação e caracterização de aglomerações produtivas. Surgiram diversas abordagens sobre aglomerações de empresas e obviamente, diversas formas de analisá-las. Dentre elas, destacam-se: o modelo formalizado por Krugman (1998), a abordagem da economia de empresas, na qual se destaca Porter (1998), as discussões sobre os clusters, com Scott (1988), da economia de inovação com a contribuição destacada de Audrestch (1988), e a abordagem de pequenas empresas, distritos industriais, com destaque de Schmitz (1994), dentre várias outras.

Os municípios selecionados situam-se no Estado do Rio de Janeiro que se encontra na região sudeste do Brasil. Este Estado ocupa uma extensão territorial aproximada de $43.780 \mathrm{Km}^{2}$ e abrigando uma população de 15.989.929 habitantes (segundo dados do IBGE, 2010); o Estado do Rio de Janeiro é geopoliticamente dividido em 92 municípios distribuídos em 18 microrregiões. De acordo com os resultados definitivos da RAIS em 2009, este detinha a $6^{\mathrm{a}}$ posição no ranking nacional de números de empregos gerados pela indústria de transformação, representando 5,37\% do total. É a segunda unidade da federação em termos de Produto Interno Bruto ( $\mathrm{R} \$$ 343.182 milhões), apresentou em 2008, crescimento de $4,1 \%$, apesar da crise internacional. Este resultado foi menor do que o nacional, que alcançou 5,2\%.

O Estado respondeu em 2008 por 11,3\% do PIB do país, sendo superado apenas por São Paulo $(33,1 \%)$, e seguido por Minas Gerais $(9,3 \%)$. Sua taxa de crescimento industrial em 2008 teve expansão de $2,7 \%$, mostrando desempenho superior ao alcançado em $2007(+0,5 \%)$. A indústria de transformação, que representa $9,9 \%$ do valor adicionado (VA), teve queda de volume de $0,8 \%$, fruto do desempenho negativo em sete ramos industriais, com destaque para as atividades "alimentos e bebidas" (-1,7\%), "têxteis" (-1,4\%), "refino de petróleo e álcool" $(-0,4 \%)$, "farmacêuticos" $(-9,0 \%)$, "produtos químicos" $(-8,0 \%)$, "perfumaria" ($8,0 \%)$, "metalurgia básica" $(-6,0 \%)$. Os que apresentaram crescimento foram: "automóveis" $(+17,7 \%)$, "caminhões e ônibus" $(+15,2 \%)$ e "cimento" $(+14,9 \%)$.

A questão em análise corresponde à relevância que os aglomerados produtivos têm no desenvolvimento local e no fomento da atividade econômica. Com isso, propõe-se analisar, verificar e avaliar as contribuições que os aglomerados produtivos oferecem às cidades em estudo. Definiu-se como objetivo levantar e constatar a relevância do aglomerado produtivo no desenvolvimento local nos municípios de Petrópolis, Angra dos Reis e Campos de Goytacazes.

Para alcançar o objetivo principal, o trabalho adotou a metodologia de levantamento bibliográfico focando no método de avaliação de cluster proposta por Suzigan et al (2003) e Britto e Albuquerque (2002), usando o quociente locacional, culminando com a importância reduzida, elevada ou NS (não significante). A metodologia é composta de sete (07) etapas, amplamente explicada.

Pode-se afirmar que os aglomerados produtivos trazem grandes benefícios para os municípios, a sociedade, às empresas e contribuem para o desenvolvimento equilibrado da cidade.

\section{REFERENCIAL TEÓRICO SOBRE OS AGLOMERADOS PRODUTIVOS}

A experiência bem sucedida da chamada Terceira Itália ampliou o interesse internacional nos estudos dos aglomerados produtivos. Nesta região havia um grande número de pequenas empresas, tornavam-se capazes de se desenvolverem rapidamente, e se estabelecerem em mercados tidos como tradicionais, exportarem produtos e criarem novos empregos dando assim, oportunidades de trabalho, 
além da minimização de custos (Humphrey; Schmitz, 1995). Graças à união delas para alcançarem o desenvolvimento desta região, que hoje é motivo de estudos.

A aglomeração é uma estratégia que as pequenas e médias empresas (PME's) utilizam para enfrentar o desafio de permanecerem atuantes no mercado. Critérios como a aproximação entre elas e o tamanho ou poder econômico/financeiro fazem com que se unam e adquiram vantagens competitivas, como economias de escala e escopo, adotando a desverticalização e especialização da produção. As empresas ao se unirem para fortalecerem suas atividades ganham o nome de aglomerados industriais (industrial clusters) ou arranjos produtivos locais (APL) (Lastres et al., 2002). Com isso, elas criam sinergia que lhes permite alcançar sucesso maior no processo/cadeia produtivo.

Os atributos socioeconômicos, institucionais e culturais, e também, a orientação do sistema de coordenação determinam a conformação de diferentes tipos de sistemas produtivos locais (Borin, 2005). Pelo que o arranjo produtivo dependerá intrinsicamente do tipo de atividades que seus integrantes desenvolvem.

Em geral, os aglomerados produtivos são formados de forma espontânea, pelo que, é possível observar regiões com características necessárias e condições favoráveis para a existência dos aglomerados, mas não há a presença dos mesmos. Mostrando que o surgimento dos aglomerados produtivos não tem sido todo planejado, mas sim, como uma necessidade de sobrevivência e competitividade das empresas. Os pontos em comuns, a boa convivência e até às debilidades detectadas nas empresas acabam sendo os fatores determinantes para a troca de informações, união e o compartilhamento de atividades entre elas, com predominância das relações informais (Neumann; Hexsel; Balestrin, 2011).

Segundo Barquero (2001), uma rede (como é o caso dos aglomerados produtivos) pode ser definida como o sistema de relações que vinculam os agentes econômicos entre si e cujo conteúdo está relacionado a bens materiais, informação ou tecnologia. Economicamente, as redes são formadas de modo geral por empresas, as quais buscam estabelecer relações avançadas com seus fornecedores, clientes e colaboradores. As redes são perfeitas para a difusão de inovações e conhecimento, assim como, a superação de deficiências produtivas.

A figura 1 resume de forma sucinta os atributos que os aglomerados de uma determinada região devem apresentar. É importante destacar a participação dos governos regionais e locais, visto que o escoamento de produtos necessita de infraestrutura e apoio do governo.

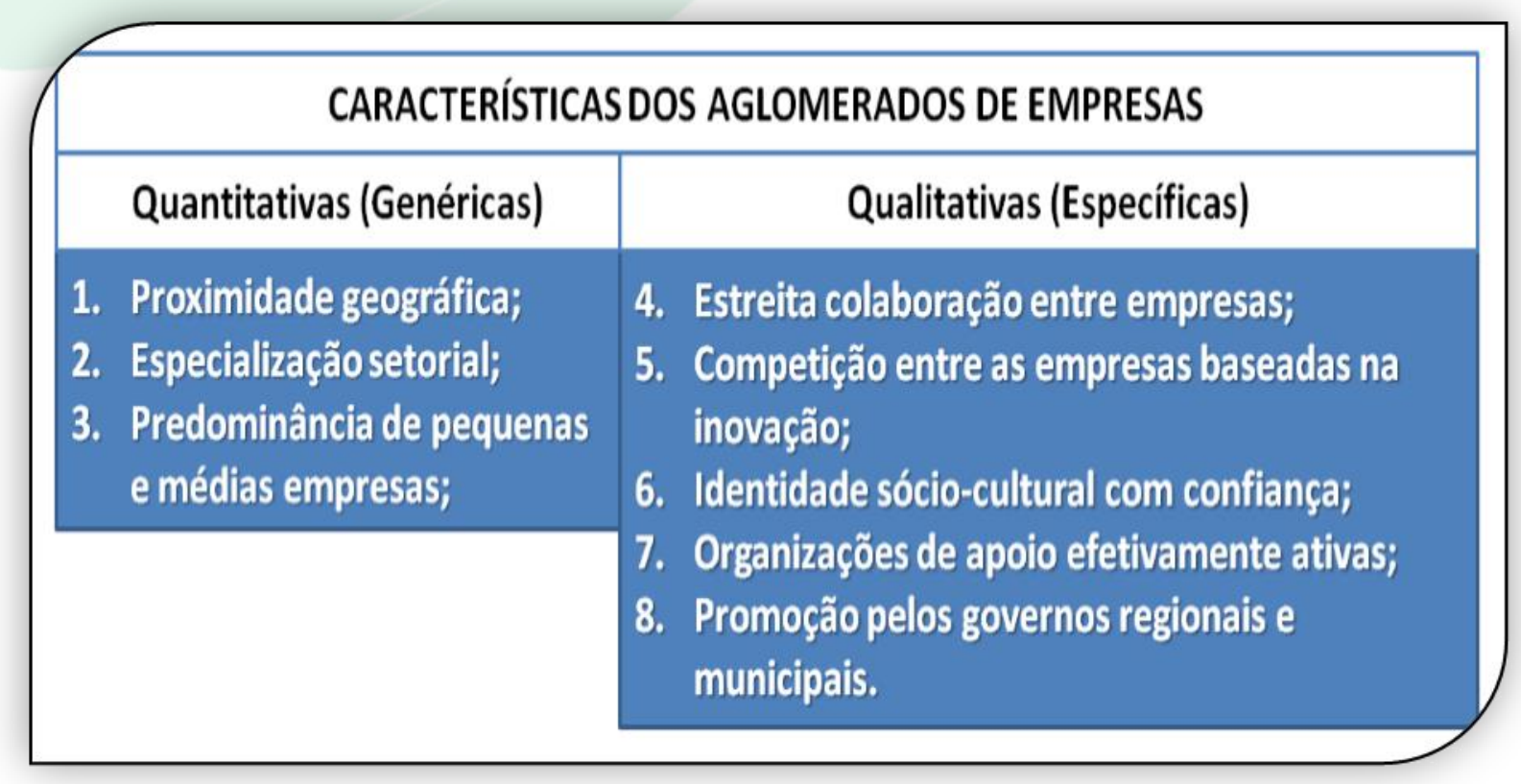

Figura 1 - Características dos aglomerados produtivos Adaptado de Schmitz (2005)

Outra visão para caracterizar aglomerados produtivos é apresentada por Stamer (2001), existem fatores de localização objetivos e subjetivos que contribuem para a existência de um aglomerado. Quando ao objetivo, encontram-se: a posição geográfica em relação aos mercados de compra e venda; ligação à rede de transportes (rodovias, ferrovias, portos, aeroportos); oferta de mão-de-obra (qualificada e adequada, nível salarial); disponibilidade de terrenos; custos com energia e meio ambiente; 
Análise dos Aglomerados Produtivos nos Municípios de Angra dos Reis, Campos e Petrópolis no Estado do Rio de Janeiro

encargos municipais; vantagens financeiras oferecidas pela cidade ou estado (incentivos fiscais, subvenções, etc.). Enquanto que o subjetivo se destaca: o ambiente econômico da cidade e da região correspondente; imagem da cidade/região; contatos setoriais; universidades, instituições de pesquisa e tecnologia; perfil inovador da região; desempenho de associações comerciais e industriais; qualidade residencial e de seu entorno; qualidade do meio ambiente; qualidade das escolas e de outras instituições de formação; qualidade da infraestrutura e qualidade e diversidade do lazer.

Os aglomerados produtivos evoluem ao longo do tempo, podendo apresenta quociente diferente ao longo dos anos e sua importância para a cidade mudar e, por isso, é preciso entender o desenvolvimento da atividade econômica e a maneira como isso ocorre em cada período de tempo (Possas, 1999).

\section{METODOLOGIA}

\subsection{Avaliação de Aglomerados}

A base metodológica adotada para a avaliação e classificação dos aglomerados produtivos é a proposta por Suzigan et al (2003) e por Britto e Albuquerque (2002). A abordagem de Suzigan resultou em uma aplicação abrangente, pois mapeou os aglomerados produtivos por unidade da federação dividida por suas microrregiões e, em relação ao extrato de atividade econômica, obviamente dentro do setor de indústria de transformação, limitou-se à Divisão CNAE (Classificação Nacional de Atividades Econômicas), não permitindo uma caracterização setorial mais específica ou detalhada. Em relação à abordagem metodológica de Britto e Albuquerque o resultado é inverso, nessa os aglomerados produtivos são identificados em um menor nível de desagregação territorial, chegando ao nível de municípios e, igualmente, mantendo o extrato de atividade econômica no nível de Divisão CNAE. Isso permite uma análise mais incisiva e pormenorizada da aglomeração em relação à abordagem metodológica de Suzigan et al (2003).

Na Figura 2, abaixo, é possível averiguar que Suzigan et al (2003) não desagregam totalmente as dimensões: territorial e setorial, enquanto Britto e Albuquerque chegam ao menor nível de desagregação apenas na dimensão territorial. A desagregação tornase mais relevante a partir do momento em que se deseja estudar a influência de uma aglomeração produtiva no desenvolvimento local. Assim conhecer mais especificamente o setor da economia e o município em que as empresas desse setor estão localizadas, na prática, torna a identificação, a caracterização e o mapeamento mais precisos.

Dentro desse contexto, apresenta-se a abordagem de avaliação proposta pelo estudo, utilizando parcialmente os procedimentos metodológicos originais de Suzigan et al. e de Britto e Albuquerque para construir os procedimentos que possam ser aplicados em níveis máximos de desagregação territorial e setorial, permitindo encontrar de forma mais acurada as vocações econômicas das cidades fluminenses. Com isso, analisa-se os grupos e até os subgrupos da CNAE para que se possa ter uma ideia clara sobre os grupos de atividades relevantes para o município e que contribuem significativamente para o desenvolvimento da cidade, tanto em nível de emprego como em ações de desenvolvimento estratégico das empresas.

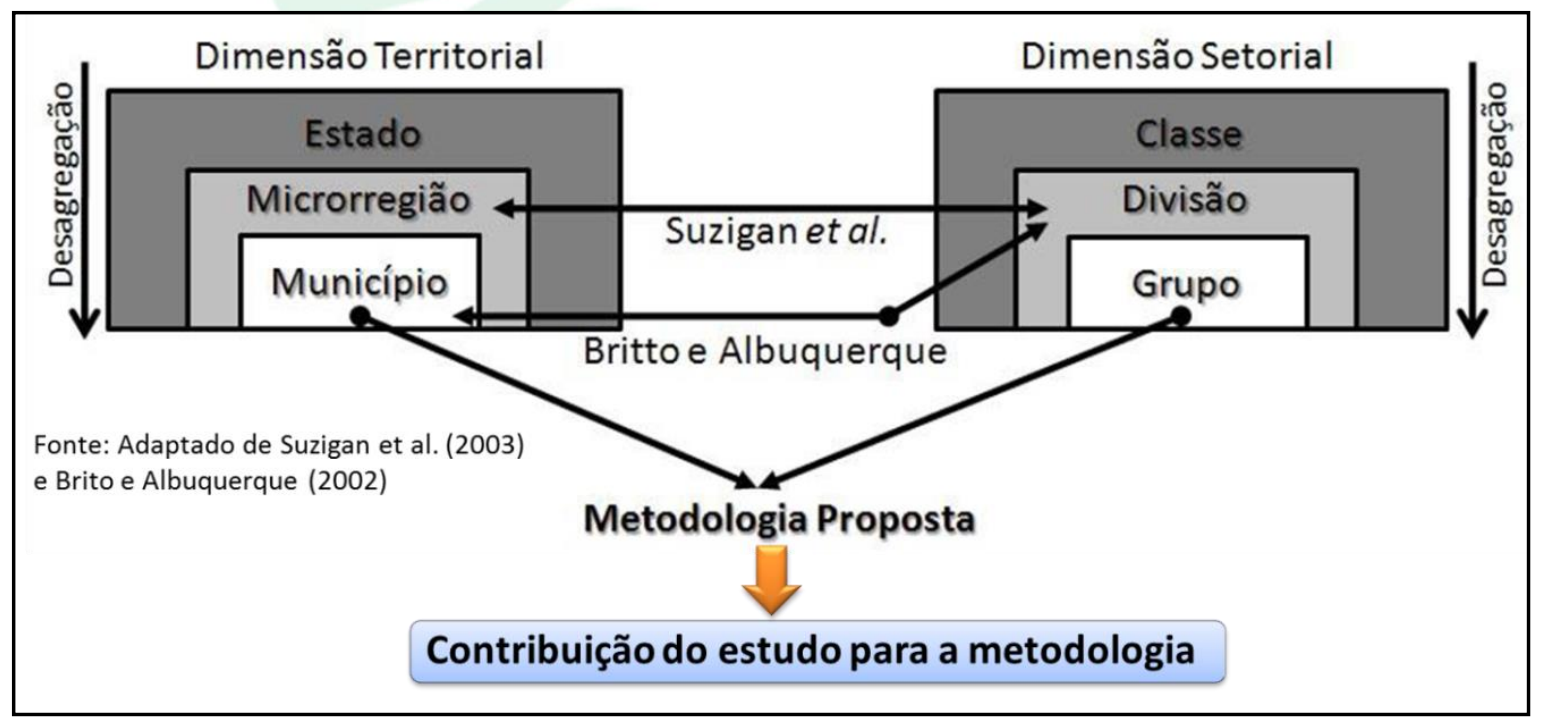

Figura 2 - Origem da abordagem proposta 


\subsection{Procedimentos da Pesquisa}

A abordagem proposta foi dividida em sete etapas sequenciais que ao final resulta em uma classificação do grau de contribuição de aglomerados produtivos, desagregados em nível de município e grupo de atividades econômicas, em relação aos municípios onde se localizam. As etapas e procedimentos que configuram a abordagem metodológica são apresentados a seguir:

- $\quad \mathbf{1}^{\text {a }}$ Etapa: Coletar dados sobre o número de empregos dos 92 municípios do Estado do Rio de Janeiro para as 24 divisões CNAE (da Divisão 10 até a Divisão 33) cuja fonte é a RAIS (Relação Anual de Informações Sociais), dos anos-base de 1999, 2004 e 2009. Da extração geral, foram recortados os três municípios abordados neste estudo.

- $2^{\text {a }}$ Etapa: Calcular o Quociente Locacional (QL) por município-divisão, utilizando o estado como região padrão ou de referência;

O Quociente Locacional (QL) mostra a setorização produtiva da região em cada uma das divisões de atividades. $\mathrm{O}$ indicador de localização ou de especialização (setorização) indica, portanto, a concentração relativa de uma determinada divisão numa região, comparativamente à participação dessa mesma divisão no espaço definido como padrão, como, no caso deste trabalho, o Estado do Rio de Janeiro. Assim, a verificação de um QL elevado em determinada atividade em uma região indica o grau de setorização da estrutura de produção local naquela atividade.

Pode-se traduzir o índice do Quociente Locacional na razão entre o número de empregos gerados de uma determinada atividade ' $\mathrm{s}$ ' em um município ' $\mathrm{m}$ ' sobre o número total de emprego desse município, representando o numerador da equação e o número total de empregos gerados dessa atividade 's' no estado ' $R$ ' sobre o total de emprego do estado, representando o denominador.

Caso o QL seja maior ou igual a um (1) indica a existência de setorização da atividade econômica no município, isto é, a atividade econômica é considerada significativamente importante na geração de empregos para a região, logo, deverá ser considerada relevante para o desenvolvimento local.

A fórmula a seguir sintetiza o cálculo do QL aplicado à variável emprego:
$Q L=\frac{\frac{E_{g m}}{E_{m}}}{\frac{E_{g R}}{E_{R}}}$
Onde:

Esm - número de emprego do setor no município;

Em - número de emprego total do município;

EsR - número de emprego do setor no estado;

ER - número de emprego total do estado.
- $\quad 3^{a}$ Etapa: Calcular o percentual de emprego por município-divisão em relação ao total do setor da região padrão (Estado);

- $4^{\text {a }}$ Etapa: Coletar dados do número de estabelecimentos para cada município-divisão do Estado do Rio de Janeiro referentes à indústria de transformação;

- $\quad 5^{a}$ Etapa: Aplicar a abordagem metodológica proposta por Suzigan et al. (2003) para classificar as aglomerações produtivas quanto à importância (Elevada ou Reduzida) para o desenvolvimento local dos municípios fluminenses em 1999, 2004 e 2009;

- $\quad 6^{\mathbf{a}}$ Etapa: Desagregar as divisões em grupos de atividades cujas aglomerações foram classificadas como tendo uma evolução igual ou superior no grau de importância para o desenvolvimento local ao longo da década.
Dessa forma, identifica-se mais precisamente qual (ais) grupo(s) de atividades econômicas, pertencentes àquela divisão, caracteriza especificamente o aglomerado;

- $7^{\text {a }}$ Etapa: Avaliar a divisão do trabalho da aglomeração para verificar sua estrutura. A presença de empresas consideradas grandes distribuídas ao longo de grupos de atividades econômicas correlacionadas induz a formação de aglomerações verticais e a presença maciça de empresas de pequeno porte que ocupam a mesma posição na cadeia de valor leva à formação de aglomerações horizontais, segundo Britto e Albuquerque (2002). Esta tese apenas indicará a possível formação vertical ou horizontal das aglomerações, pois a exata classificação necessitaria de análises mais aprofundadas sobre a presença de indústrias de bens de capital, comércios, atacadistas e varejistas, assim como a 
Análise dos Aglomerados Produtivos nos Municípios de Angra dos Reis, Campos e Petrópolis no Estado do Rio de Janeiro

evidenciação da existência da correlação entre eles; o que acaba se desviando do foco proposto.
$\mathrm{Na}$ figura que se segue, encontra-se resumida as sete etapas descritas acima.

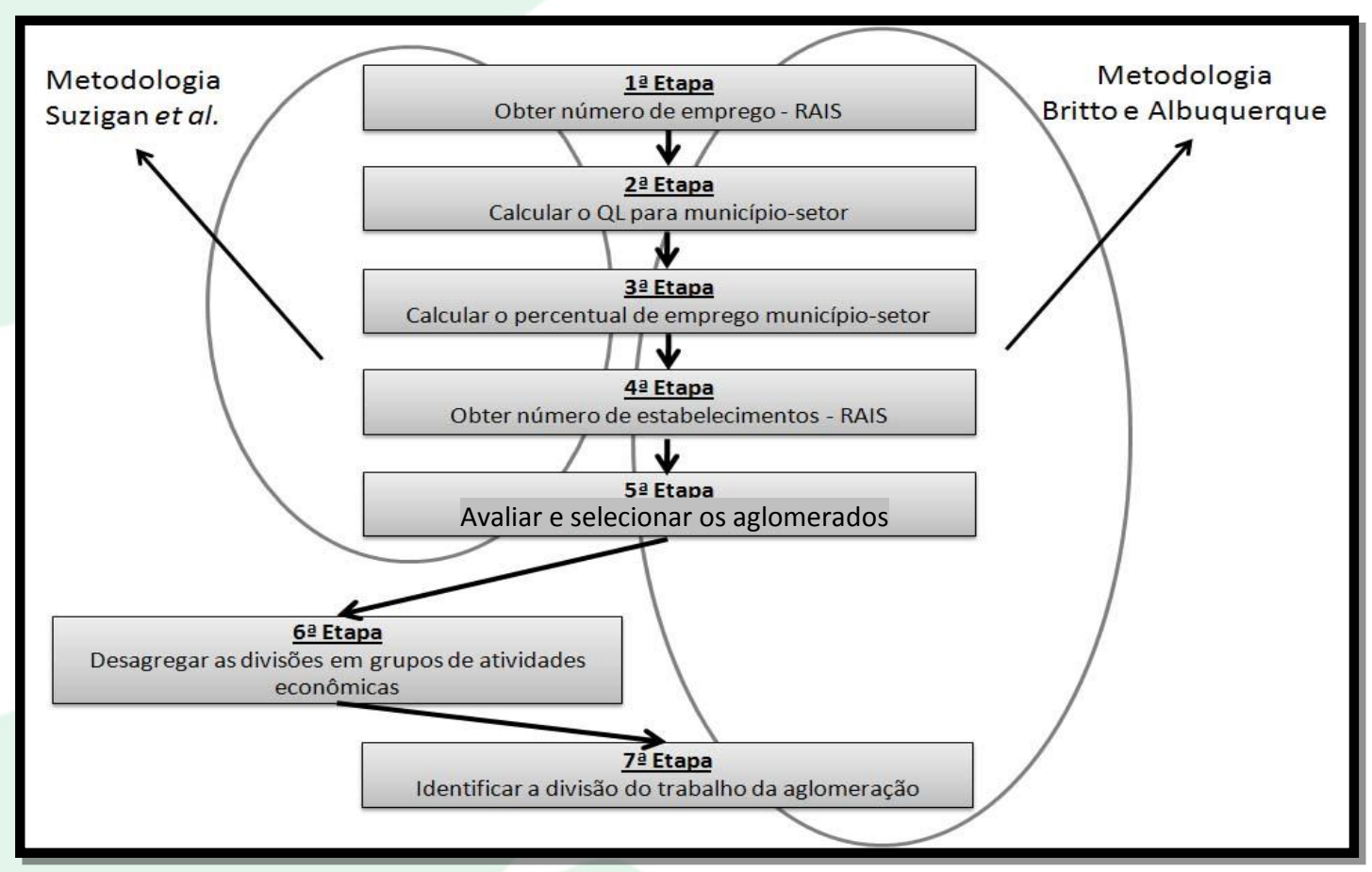

Figura 3 - Etapas e contribuições de Suzigan et al. (2003) e Britto e Albuquerque (2002) para a formulação da abordagem de avaliação

\section{RESULTADO E DISCUSSÃO}

\subsection{Avaliação dos Aglomerados Produtivos das Três Cidades em 1999, 2004 e 2009}

De acordo com a abordagem metodológica proposta por Suzigan et al. (2004), as aglomerações possuem dois tipos de contribuição ou importância para desenvolvimento local - importância elevada ou importância reduzida, sendo o valor do QL o principal responsável por essa classificação. Ainda, para efeito deste trabalho, estabeleceu-se a classe Não Significativa (NS) para as aglomerações de empresas que apresentarem importância abaixo de reduzida. Em resumo, como ilustrado pela Tabela 1, o aglomerado que apresentar um QL igual ou superior a 05 (cinco), ter um percentual de emprego (\% Emp.) igual ou maior que 1,00 (um) e 05 (cinco) ou mais estabelecimentos é classificado como tendo uma importância elevada para o local. 
Análise dos Aglomerados Produtivos nos Municípios de Angra dos Reis, Campos e Petrópolis no Estado do Rio de Janeiro

Tabela 1 - Resultado da classificação de aglomerados - 2009

\begin{tabular}{|c|c|c|c|c|c|c|c|c|c|c|c|c|}
\hline \multirow{2}{*}{$\begin{array}{c}\text { Divisão } \\
\text { CNAE 2.0 }\end{array}$} & \multicolumn{4}{|c|}{ ANGRA DOS REIS } & \multicolumn{4}{|c|}{ CAMPOS DOS GOYTACAZES } & \multicolumn{4}{|c|}{ PETRÓPOLIS } \\
\hline & $\mathbf{Q L}$ & \%Еmp & $\mathbf{N}^{0}$. Est. & Imp. & $\mathbf{Q L}$ & \%Emp & $\mathrm{N}^{\circ}$. Est. & Imp. & $\mathbf{Q L}$ & \%Emp & $\mathrm{N}^{\circ}$.Est. & Imp. \\
\hline 10 & 0,03 & 0,07 & 7 & NS & 3,47 & 8,40 & 74 & Reduzida & 0,45 & 1,86 & \begin{tabular}{|l|l|}
75 \\
\end{tabular} & NS \\
\hline 11 & 0,00 & 0,00 & 0 & NS & 0,24 & 0,58 & 5 & NS & 1,63 & 6,76 & 4 & NS \\
\hline 12 & 0,00 & 0,00 & 0 & NS & 0,99 & 2,41 & 1 & NS & 0,00 & 0,00 & 0 & NS \\
\hline 13 & 0,06 & 0,15 & 2 & NS & 0,27 & 0,65 & 8 & NS & 5,05 & 20,97 & 56 & Elevada \\
\hline 14 & 0,02 & 0,05 & 13 & NS & 0,40 & 0,97 & 54 & NS & 2,33 & 9,67 & 359 & Reduzida \\
\hline 15 & 0,00 & 0,00 & 0 & NS & 0,65 & 1,58 & 5 & NS & 0,36 & 1,51 & 7 & NS \\
\hline 16 & 2,28 & 5,23 & 4 & NS & 0,24 & 0,58 & 2 & NS & 1,13 & 4,69 & 17 & Reduzida \\
\hline 17 & 0,00 & 0,00 & 0 & NS & 0,07 & 0,18 & 5 & NS & 0,71 & 2,94 & 10 & NS \\
\hline 18 & 0,16 & 0,36 & 7 & NS & 0,43 & 1,03 & 20 & NS & 1,00 & 4,14 & 23 & NS \\
\hline 19 & 0,00 & 0,00 & 0 & $\mathrm{NS}$ & 0,00 & 0,00 & 0 & NS & 0,00 & 0,00 & 0 & NS \\
\hline 20 & 0,02 & 0,04 & 2 & NS & 0,33 & 0,79 & 4 & NS & 0,01 & 0,03 & 4 & NS \\
\hline 21 & 0,00 & 0,00 & 0 & $\mathrm{NS}$ & 0,33 & 0,79 & 2 & NS & 0,00 & 0,00 & 0 & NS \\
\hline 22 & 0,00 & 0,00 & 0 & NS & 0,19 & 0,46 & 11 & NS & 0,91 & 3,79 & 44 & NS \\
\hline 23 & 0,04 & 0,09 & 5 & NS & 5,67 & 13,74 & 155 & Elevada & 0,27 & 1,12 & 30 & NS \\
\hline 24 & 0,03 & 0,07 & 3 & NS & 0,47 & 1,13 & 3 & NS & 0,06 & 0,23 & 3 & NS \\
\hline 25 & 0,45 & 1,04 & 10 & NS & 0,29 & 0,69 & 29 & NS & 0,56 & 2,32 & 39 & NS \\
\hline 26 & 0,00 & 0,00 & 0 & NS & 0,00 & 0,00 & 0 & NS & 1,33 & 5,51 & 7 & Reduzida \\
\hline 27 & 0,23 & 0,52 & 2 & NS & 0,23 & 0,56 & 2 & NS & 0,16 & 0,66 & 3 & NS \\
\hline 28 & 0,00 & 0,00 & 0 & NS & 0,12 & 0,29 & 7 & NS & 0,42 & 1,76 & 17 & NS \\
\hline 29 & 0,09 & 0,21 & 5 & NS & 0,53 & 1,28 & 10 & NS & 0,05 & 0,19 & 2 & NS \\
\hline 30 & 14,20 & 32,59 & 10 & Elevada & 0,03 & 0,08 & 1 & NS & 0,00 & 0,00 & 1 & NS \\
\hline 31 & 0,00 & 0,00 & 0 & NS & 1,42 & 3,43 & 16 & Reduzida & 1,70 & 7,07 & 57 & Reduzida \\
\hline 32 & 0,00 & 0,00 & 0 & NS & 0,14 & 0,34 & 10 & NS & 3,98 & 16,53 & 25 & Reduzida \\
\hline 33 & 0,69 & 1,59 & 15 & NS & 0,88 & 2,13 & 23 & NS & 1,66 & 6,89 & 13 & Reduzida \\
\hline
\end{tabular}

Fonte: Elaborada pelos autores.

Aquele cujo QL for maior ou igual a 01 (um) e QL menor que 05 (cinco), também com percentual de emprego (\% Emp.) igual ou maior que 1,00 (um) e 05 (cinco) estabelecimentos no mínimo é classificado como de importância reduzida para o local. Portanto, com a aplicação dos critérios o par município-setor é separado de acordo com sua importância na geração de empregos, contribuindo assim com sua parcela para o desenvolvimento local.

Ainda em relação à Tabela 1 , a Divisão 30 Fabricação de outros equipamentos de transporte, exceto veículos automotores (CNAE 2.0) no município de Angra dos Reis é a única que superou os critérios estabelecidos, apresentando: QL igual a 14,20; percentual de emprego de $32,59 \%$ e 10 estabelecimentos registrados no setor, sendo assim, a aglomeração apresenta uma contribuição elevada. Em relação a Campos dos Goytacazes, a Divisão 31 Fabricação de móveis QL intermediário de 1,42; percentual de $3,43 \%$ e número de estabelecimentos igual a 16, logo, classificada como reduzida. Aquelas que não conseguiram vencer os crivos estabelecidos são classificadas como não significativas.
A $6^{\text {a }}$ Etapa permite avaliar a dinâmica de evolução das aglomerações produtivas ao longo do tempo. Em termos de resultados, foram identificadas 2.116 aglomerações (92 municípios e 23 atividades) em 1999, 2.116 aglomerações em 2004 e 2.208 aglomerações (92 municípios e 24 atividades) em 2009, totalizando 6.640 aglomerados de empresas.

Analisando a Tabela 2, percebe-se que algumas aglomerações, em relação ao grau de contribuição para o Desenvolvimento Local (DL) cresceram ao longo dos 10 anos (1999-2009), por exemplo: fabricação de outros equipamentos de transporte em Angra dos Reis; fabricação de produtos têxteis em Petrópolis. Outras se mantiveram estáveis como os aglomerados de fabricação de produtos alimentícios do município de Campos, confecção de artigos do vestuário e acessórios e fabricação de móveis de Petrópolis; finalmente algumas decresceram ao longo da década, citando, com o exemplo, fabricação de celulose, papel e produtos de papel também do município de Petrópolis. 
Análise dos Aglomerados Produtivos nos Municípios de Angra dos Reis, Campos e Petrópolis no Estado do Rio de Janeiro

Tabela 2 - Aglomerações que apresentaram significativa importância em pelo menos um dos anos-base.

\begin{tabular}{|c|c|c|c|c|c|}
\hline \multicolumn{3}{|r|}{ MUNICÍPIO: ANGRA DOS REIS } & \multicolumn{3}{|c|}{ Importância para o DL } \\
\hline Divisão 1.0 & Divisão 2.0 & Descrição da Atividade Econômica & 1999 & 2004 & 2009 \\
\hline 35 & 30 & Fabricação de outros equipamentos de transporte, exceto veículos automotores. & NS & Elevada & Elevada \\
\hline \multicolumn{3}{|r|}{ MUNICÍPIO: CAMPOS DOS GOYTACAZES } & \multicolumn{3}{|c|}{ Importância para o DL } \\
\hline Divisão 1.0 & Divisão 2.0 & Descrição da Atividade Econômica & 1999 & 2004 & 2009 \\
\hline 15 & 10 & Fabricação de produtos alimentícios & Reduzida & Reduzida & Reduzida \\
\hline 19 & 15 & Preparação de couros e fabricação de artefatos de couro... & Reduzida & NS & NS \\
\hline 26 & 23 & Fabricação de produtos de minerais não metálicos & Elevada & Reduzida & Elevada \\
\hline 36 & 31 & Fabricação de móveis & Reduzida & NS & Reduzida \\
\hline \multicolumn{3}{|r|}{ MUNICÍPIO: PETRÓPOLIS } & \multicolumn{3}{|c|}{ Importância para o DL } \\
\hline Divisão 1.0 & Divisão 2.0 & Descrição da Atividade Econômica & 1999 & 2004 & 2009 \\
\hline 17 & 13 & Fabricação de produtos têxteis & Reduzida & Reduzida & Elevada \\
\hline 18 & 14 & Confecção de artigos do vestuário e acessórios & Reduzida & Reduzida & Reduzida \\
\hline 20 & 16 & Fabricação de produtos de madeira & NS & NS & Reduzida \\
\hline 21 & 17 & Fabricação de celulose, papel e produtos de papel & Reduzida & Reduzida & NS \\
\hline 30 & 26 & Fabricação de equipamentos de informática, produtos eletrônicos e opt. & NS & NS & Reduzida \\
\hline 36 & 31 & Fabricação de móveis & Reduzida & Reduzida & Reduzida \\
\hline 33 & 33 & Manutenção, reparação e instalação de máquinas e equipamentos. & NS & NS & Reduzida \\
\hline
\end{tabular}

Fonte: Elaborada pelos autores.

Para efeito de simplificação, os aglomerados produtivos que apresentaram contribuição crescente ou mantiveram-se na classe elevada são chamados aqui de aglomerações de variação positiva ou de experiência positiva para o desenvolvimento local. As diversas combinações que representam as aglomerações com experiência positiva são apresentadas na Tabela 3 .
No total, foram 29 aglomerações produtivas (09 do Grupo A, 05 do Grupo B, 06 do Grupo C, 01 do Grupo D e 08 do Grupo E). Similarmente, considerando a amostra utilizada nesta seção, a relação de aglomerados por grupos é apresentada na Tabela 3.

Tabela 3 - Aglomeração com experiência positiva.

\begin{tabular}{|c|c|c|c|}
\hline \multirow{2}{*}{ GRUPOS } & \multicolumn{3}{|c|}{ IMPORTÂNCIA } \\
\cline { 2 - 4 } & 1999 & 2004 & 2009 \\
\hline A & Elevada & Elevada & Elevada \\
\hline B & NS & Elevada & Elevada \\
\hline C & Reduzida & Reduzida & Elevada \\
\hline D & NS & Reduzida & Elevada \\
\hline E & NS & NS & Elevada \\
\hline
\end{tabular}

Fonte: Elaborada pelos autores.

A aglomeração produtiva do setor de fabricação de produtos de minerais não metálicos (Divisão 26) da cidade de Campos dos Goytacazes não consta nessa relação, pois, curiosamente, foi classificada em 2004, como tendo importância reduzida por não ter atingido um QL mínimo de 05 (vide Tabela $1)$.

Tabela 4 - Relação de aglomerados por extratos de variação positiva.

\section{MUNICÍPIO: ANGRA DOS REIS}

\begin{tabular}{|c|c|l|l|}
\hline Divisão 1.0 & Divisão 2.0 & Descrição da Atividade Econômica & \multicolumn{1}{|c|}{ MUNICÍPIO: PETRÓPOLIS } \\
\hline 35 & 30 & Fabricação de outros equipamentos de transporte, exceto veículos automotores. \\
\hline \multicolumn{3}{|c|}{ Gupo B } \\
\hline Divisão 1.0 & Divisão 2.0 & Descrição da Atividade Econômica \\
\hline 17 & 13 & Fabricação de produtos têxteis \\
\hline
\end{tabular}

Fonte: Elaborada pelos autores. 
Após a seleção quanto à dinâmica de evolução do grau de contribuição para o desenvolvimento local, mas ainda na etapa seis da abordagem metodológica, faz-se a desagregação da divisão da atividade econômica em grupos de atividades. Esse procedimento permite conhecer mais especificamente em que "subsetor" o aglomerado produtivo é especializado.
Para ilustrar toma-se como exemplo o município de Angra dos Reis. O setor que se destacou foi o de fabricação de outros equipamentos de transporte, exceto veículos automotores (Divisão 30 CNAE 2.0), aplicando a desagregação de divisão para grupos de atividades econômicas percebe-se que o tipo de veículo de transporte fabricado, especificamente, naquela cidade (Tabela 5).

Tabela 5 - Desagregação da Divisão 30 de atividades econômicas do município de Angra dos Reis

\begin{tabular}{|c|c|c|c|c|}
\hline \multicolumn{5}{|c|}{ Número de empregos } \\
\hline Grupo 2.0 & Descrição da Atividade Econômica & 1999 & 2004 & 2009 \\
\hline 30.1 & Construção de embarcações & 24 & 5.318 & 7.112 \\
\hline 30.3 & Fabricação de veículos ferroviários & 0 & 0 & 0 \\
\hline 30.4 & Fabricação de aeronaves & 0 & 0 & 0 \\
\hline 30.5 & Fabricação de veículos militares de combate & 0 & 0 & 0 \\
\hline 30.9 & $\begin{array}{l}\text { Fabricação de equipamentos de transporte não especificados } \\
\text { anteriormente }\end{array}$ & 0 & 0 & 0 \\
\hline
\end{tabular}

Fonte: Elaborada pelos autores.

Como visto na Tabela 5, indubitavelmente, a vocação econômica do município de Angra dos Reis é a indústria naval, até por que sua geografia favorece o desenvolvimento desta atividade. Por conseguinte, pode-se afirmar que existe uma aglomeração produtiva do setor de construção de embarcações em Angra dos Reis e que contribui para o desenvolvimento local através de uma significativa e crescente geração de empregos.

A sétima e última etapa proposta pelo estudo é fruto da contribuição direta do trabalho de Britto e Albuquerque. Ela visa à caracterização da estrutura produtiva do aglomerado através da análise da cadeia de valor. Caso a estrutura produtiva seja distribuída ao longo da cadeia com presença efetiva de empresas atuantes em setores correlatos, fornecendo matéria prima, máquinas e equipamentos e escoando a produção via comércio: atacadistas e varejistas tem-se aglomerações integrantes de uma rede vertical de relacionamentos. Por outro lado, caso as empresas sejam mais especializadas em um elo da cadeia de valor, as aglomerações são consideradas como integrantes de redes horizontais de relacionamentos (Britto e Albuquerque, 2002). A Tabela 6 mostra a classificação por porte de empresa.

Tabela 6 - Classificação por porte de empresa.

\begin{tabular}{|l|l|l|}
\hline IBGE & \multirow{2}{*}{ Adaptada } \\
\hline Porte & $N^{\text {o }}$ de empregados & \\
\hline Micro & Até 19 & \multirow{2}{*}{ Pequeno porte (PP) } \\
\hline Pequena & De 20 a 99 & Médio porte (MP) \\
\hline Média & De 100 a 499 & Grande porte (GP) \\
\hline Grande & Mais de 500 & \\
\hline
\end{tabular}

Fonte: Elaborada pelos autores.

A título de exemplo, retoma-se a setor de construção de embarcações em Angra dos Reis que apresenta uma estrutura produtiva em torno de uma empresa de grande porte seguida de duas de médio porte e sete de pequeno porte em 2009 , induzindo a formação de uma rede verticalizada, entretanto, como mencionado anteriormente, somente com a pesquisa empírica essa afirmação poderá ser comprovada (Tabela 7). 
Análise dos Aglomerados Produtivos nos Municípios de Angra dos Reis, Campos e Petrópolis no Estado do Rio de Janeiro

Tabela 7 - Estrutura produtiva do aglomerado do setor de construção de embarcações de Angra dos Reis.

\begin{tabular}{|c|l|c|c|c|c|c|c|c|c|c|}
\hline \multicolumn{2}{|c|}{ Número de estabelecimentos por porte } & \multicolumn{3}{|c|}{1999} & \multicolumn{3}{c|}{2004} & \multicolumn{3}{c|}{2009} \\
\hline Grupo 2.0 & Descrição da Atividade Econômica & PP & MP & GP & PP & MP & GP & PP & MP & GP \\
\hline 30.1 & Construção de embarcações & 8 & 0 & 0 & 13 & 2 & 1 & 7 & 2 & 1 \\
\hline
\end{tabular}

Fonte: Elaborada pelos autores.

A atividade de maior relevância (importância elevada) do município de Angra dos Reis durante os dez anos em estudo foi a divisão 30 fabricação de outros equipamentos de transporte, exceto veículos automotores (CNAE 2.0), ocupando a $21^{a}$ posição no ranking de atuação das aglomerações produtivas do Estado em 1999 e com a $18^{\text {a }}$ posição no ranking de 2009. Ao longo dos dez anos, esta atividade teve evolução, em 1999 sua importância era NS (não significativa), passando em 2004 e 2009 para importância elevada para o município de Angra dos Reis.

O município de Campos dos Goytacazes apresenta quatro atividades avaliadas, sendo que apenas uma a Divisão 23 - fabricação de produtos de minerais não metálicos (CNAE 2.0) apresentou importância elevada em 1999 e 2009 e reduzida em 2004. Esta atividade ocupa a $1^{\mathrm{a}}$ posição no ranking da atuação das aglomerações produtivas no Estado do Rio de Janeiro, isto em 1999 com frequência de 13 e 2009 com frequência de 16 , conservando o $1^{\circ}$ lugar do ranking.

O município de Petrópolis teve apenas uma atividade (Divisão 13 - fabricação de produtos têxteis, CNAE 2.0) avaliada com importância elevada em 2009 e, nos restantes anos se manteve com importância reduzida ou NS. A atividade com importância elevada ocupou a $12^{\text {a }}$ posição em 1999 e a $9^{a}$ posição em 2009 no ranking da atuação das aglomerações produtivas no Estado do Rio de Janeiro.

Tabela 8 - Posição dos três municípios no Estado

\begin{tabular}{|c|c|c|c|c|c|c|c|c|}
\hline \multirow{2}{*}{ Município } & \multicolumn{2}{|r|}{ CNAE } & \multicolumn{2}{|c|}{ Posição RJ } & \multicolumn{2}{|c|}{ Importância } & \multicolumn{2}{|c|}{ Frequência } \\
\hline & Divisão 2.0 & $\begin{array}{c}\text { Nome } \\
\end{array}$ & 1999 & 2009 & 1999 & 2009 & 1999 & 2009 \\
\hline $\begin{array}{l}\text { Angra dos } \\
\text { Reis }\end{array}$ & 30 & $\begin{array}{l}\text { Fabricação de outros equipamentos de } \\
\text { transporte, exceto veículos automotores. }\end{array}$ & $21^{\mathrm{a}}$ & $18^{\mathrm{a}}$ & NS & Elevada & 01 & 02 \\
\hline $\begin{array}{l}\text { Campos dos } \\
\text { Goytacazes }\end{array}$ & 23 & $\begin{array}{l}\text { Fabricação de produtos de minerais não } \\
\text { metálicos. }\end{array}$ & $1^{\mathrm{a}}$ & $1^{\mathrm{a}}$ & Elevada & Elevada & 13 & 16 \\
\hline Petrópolis & 13 & Fabricação de produtos têxteis. & $12^{\mathrm{a}}$ & $9^{a}$ & Elevada & Elevada & 04 & 6 \\
\hline
\end{tabular}

Fonte: Elaborada pelos autores.

Dez anos depois, mais 13 aglomerados produtivos surgiram no estado, 30 com importância elevada, significando um aumento de $09 \mathrm{em}$ comparação com o ano de 1999, e mais 05 com importância reduzida, perfazendo um total geral de 122 aglomerações fluminenses para o ano de 2009. A Divisão 23 , fabricação de produtos de minerais não metálicos manteve sua superioridade, sendo ajudada com um saldo positivo de 03 novas aglomerações: 07 novas (Araruama, Belford Roxo, Cantagalo, Pinheiral, Rio das Ostras, Seropédica e Tanguá) e 04 dissociadas (Barra do Piraí, Magé, Resende e São Gonçalo).

3.2 Relevância e benefícios dos aglomerados produtivos das trêsOs resultados do levantamento sobre os aglomerados produtivos revelam que algumas atividades são de extrema importancia para as cidades e geram várias oportunidade de emprego. A tabela 8 mostra a relevância e variabilidade das atividades nos municípios. Se o número de atividades economicas impactantes no município aumentarem, o que revela a presença de forte concentração de empresas, o município acaba tendo forte atividade econômica e aumenta sua importância no Estado, na geração de emprego e na produção.

Os benefícios de aglomerados produtivos para o município são vários, desde infraestrutura até a competitividade do mercado e melhoria significativa das ações econômicas. Pode-se destacar as contribuições de Vasconcelos et al (2005) que resumidamente aponta como principais benefícios dos aglomerados produtivos o ambiente institucional, economia em custos de transação (potencializada pela proximidade entre as empresas e a facilidade e velocidade de comunicação em um limitado espaço geográfico), benefícios não pecuniários, knowledge spillover (efeito da transposição do conhecimento tácito ou não codificado para além das fronteiras da empresa).

Adicionalmente, outros benefícios são visíveis com a a presença de aglomerados produtivos na aposta do desenvolvimento local, como: 
- Sociais: os aglomerados proporcionam oportunidades de emprego que concomitantemente ajudam a aumentar o nível e a qualidade de vida dos moradores. Este benefício está ligado à relação empresas e população local.

- Mercadológicos - vantagem competitiva: a junção de forças entre as empresas para formar um único grupo proporciona facilidades de interação entre elas e o mercado, permitindo deste modo ter capacidade para enfrentar os concorrentes e manter seu produto/serviço competitivo em função do seu diferencial e da rede que o assiste.

- Estrutural (alta mobilidade urbana): quando o governo oferece apoio para o fomento dos aglomerados produtivos pretende com isso melhorar e aumentar a infraestrutura da cidade e, com isso, criar mobilidade que permita integrar a cidade aos grandes centros e proporcionar ao cidadão ocasião de adquiuir localmente produtos e serviços de qualidade. Às ações de desenvolvimento das aglomerações devem ir de acordo com a política de governo para facilitar a mobilidade.

Os aglomerados identificados, avaliados e classificados nas cidades de Petropólis, Campos e Angra dos Reis têm contribuído para que o município possa apresentar forma de desenvolvimento equilibrado, porém, esse assunto é bastante envolvente e merece a atenção de todos, desde moradores até poder político, passando pelas empresas sediadas no município. Ao longo da série de anos em estudo, contemplou-se que às atividades não são estáticas, mais dinâmicas. Pelo que, sua importância para o município estará intrinsicamente ligada às ações e planos que quer o governo local quer às empresas colocam em prática.

\section{CONSIDERAÇÕES FINAIS}

Os aglomerados produtivos locais estão ocupando cada vez mais lugar estratégico no desenvolvimento das cidades e no desempenho e posicionamento estratégico das empresas. Suas múltiplas vantagens beneficiam as organizações, a sociedade e os governos locais. O desenvolvimento local equilibrado faz com que todas às áreas sejam beneficiadas pela existência de aglomerações produtivas que permite reduzir a insatisfação do cliente na busca por bens ou serviços que atendam às suas necessidades e desejos e ter melhoria contínua no nível de serviço e na satisfação das exigências do consumidor.
Este estudo procurou aplicar a metodologia proposta para avaliação e classificação de aglomerados nos municípios de Angra dos Reis, Campos e Petrópolis, de modo a observar como estes aglomerados ajudam nas atividades comerciais da cidade e contribuem para a construção de uma cidade cada vez mais desenvolvida e que atenda as expetativas de seus habitantes. Importa salientar que o processo não é isolado, mas integrado. Ou seja, inclui outros setores como marketing, produção, finança, etc.

A proposta do estudo foi alcançada e foi possível observar que as cidades apresentam características que propiciam a existência de aglomerados produtivos e que estes contribuem para o desenvolvimento da cidade e melhoria do sortido disponibilizado ao cidadão. Assim como se observou, o desenvolvimento da cidade acaba sendo uma conjugação de esforços públicos (governos) e privados (empresas) para oferecer além de bens e serviços, oportunidade de vínculo empregatício e um nível de vida melhor, acompanhado de infraestrutura aceitável social e economicamente.

Entende-se que apesar de alcançar o propósito, este trabalho não é terminal, mas o aflorar de discussões de temas ligados aos aglomerados produtivos com os demais setores empresariais. O processo de identificação e classificação dos aglomerados produtivos torna mais simples a integração sinérgica para gerar a alavancagem da atividade econômica no município, permitindo visualizar com maior precisão a localização dos polos de desenvolvimento da cidade e quais atividades econômicas devem merecer maior atenção.

Finalmente, uma das principais dificuldades em estudar aglomerações produtivas, recai na tarefa de obter dados específicos que qualifiquem de forma satisfatória essas aglomerações. Para tanto, a que dar início a um processo de mineração de dados, consultando diversos trabalhos com inúmeros objetivos de pesquisa e, quando os encontra, quase sempre já não traduzem mais a realidade atual, por estarem obsoletos.

Devido a isso, muitos estudos e pesquisas sobre aglomerados de empresas lançam mão de pesquisas empíricas para a coleta de dados diretos, o que demanda tempo e recursos consideráveis para fazer tal levantamento. Apesar das dificuldades enfrentadas, considera-se que o estudo sobre aglomerados produtivos, sua relevância para o desenvolvimento equilibrado das cidades deve continuar e se possível, estabelecer comparações entre cidades com aglomerados produtivos mais efetivos e estratégicos, assim como, analisar o papel dos governos locais ou regionais nos aglomerados produtivos e, mostrar os benefícios da existência dos aglomerados para as cidades com este modelo de desenvolvimento. 
Análise dos Aglomerados Produtivos nos Municípios de Angra dos Reis, Campos e Petrópolis no Estado do Rio de Janeiro

\section{REFERÊNCIAS}

Amato Neto, J. (2000) Redes de cooperação produtiva e clusters regionais: oportunidades para as pequenas e médias empresas. São Paulo: Atlas.

Audretsch, D. B. (1988) Agglomeration and the location of innovative activity. Oxford Review of Economic Policy, 14: 2, Summer.

Ballou, R. M. (2003) Logística empresarial: transporte, administração de materiais e distribuição física. São Paulo: Atlas.

Barquero, Antonio Vázquez. (2001) Desenvolvimento endógeno em tempos de globalização. Porto Alegre: Fundação de Economia e Estatística FEE.

Barros, Aidil J. da Silveira; Lehfeld, Neide A. de Souza. (1986) Fundamentos de metodologia científica, um guia básico para a iniciação científica. $2^{\mathrm{a}}$ Edição, Ampliada. São Paulo: Makron Books do Brasil.

Bertaglia, Paulo Roberto. (2009) Logística e Gerenciamento da Cadeia de Abastecimento. $2^{\mathrm{a}}$ edição. São Paulo: Saraiva.

Bio, Sérgio Rodrigues; Faria, Ana Cristina; Robles, Léo Tadeu. (mar/2002) Em busca da vantagem competitiva: trade-offs de custos logísticos em cadeias de suprimentos. Artigo publicado na Revista de Contabilidade CRC-SP, São Paulo, v. 6, n. 19 , p. 5-18.

Borin, E. C. P. (2005) O SEBRAE e os arranjos produtivos locais: o caso de Friburgo. In: XI Semana de Planejamento Urbano e Regional da UFRJ, 2005, Rio de Janeiro. XI Semana de Planejamento Urbano e Regional da UFRJ.

Bowersox, Donald J; Closs, David J. (2001) Logística Empresarial: O Processo de Integração da Cadeia de Suprimento. São Paulo: Editora Atlas S.A.

Britto, J.; ALBUQUERQUE, E. M. (2002) Clusters industriais na economia brasileira: uma análise exploratória a partir de dados da RAIS. Estudos Econômicos. São Paulo: v.32, n.1, p.71 - 102.

Campos, Vicente Falconi. (2004) TOC - Controle da Qualidade Total (no estilo japonês). Nova Lima MG: IND Tecnologia e Serviços Ltda.

Cassiolato, J. E.; Szapiro, M. (2003) Proposição de políticas para a promoção de sistemas produtivos locais de micro, pequenas e médias empresas,
Arranjos e sistemas produtivos locais no Brasil. Instituto de Economia da Universidade Federal do Rio de Janeiro - IE/UFRJ, Rio de Janeiro.

Christopher, Martin. (2012) Logística e Gerenciamento da Cadeia de Suprimentos. $4^{\mathrm{a}}$ ed. São Paulo: Cengage Learning.

Coyle, John J.; Bardi, Edward J.; Langley, C. John (2003) The management of business logistics: a supply chain perspective. $7^{\text {a }}$ ed. Mason, $\mathrm{OH}$ : South-western. ISBN 0-324-00751-5.

Covey, Stephen R. (2002) Liderança baseada em princípios. Rio de Janeiro: Campus.

Dessler, Gary. (2003) Administração de Recursos Humanos. $2^{a}$ edição. São Paulo: Prentice Hall.

Hindle, Tim. Tudo sobre administração São Paulo: Editora Nobel, 2002.

HUMPHREY, J.; SCHMITZ, H. (1995) Principles for Promoting Clusters and networking of SMEs. UNIDO.

Lastres, H.; Albagli, S.; Lemos, C.; Legey, L-R. (2002) Desafios e Oportunidades da Era do Conhecimento. Revista São Paulo em Perspectiva, v. 16, n. 3, p. 60-66.

Faria, Ana Cristina; Costa, Maria de Fátima Gameiro. (2008) Gestão de Custos Logísticos. $1^{a}$ edição. $3^{\mathrm{a}}$ Reimpressão. São Paulo: Atlas.

Fleury, P. F.; WANKE, Peter; Figueredo, K. F. (2000) Logística empresarial: a perspectiva brasileira. São Paulo: COPEAD-UFRJ/Atlas.

Krugman, P. (1998) What's new about the new economic geography? Oxford Review of Economic Policy, 14: 2, Summer.

Kobayashi, Shun'ichi. (2000) Renovação da Logística: como definir as estratégias de distribuição física global. $1^{a}$ edição. São Paulo: Atlas.

Kotler, Philip, Armstrong, Gary. (1998) Princípios de marketing. Rio de Janeiro: Prentice Hall do Brasil.

Neumann, L. ; Hexsel, A. ; Balestrin, Alsones. (2011) Desafios à Cooperação em Aglomerados Produtivos: Um Estudo de Caso no Segmento de Malhas do Sul do Brasil. BASE: Revista de Administração e Contabilidade da Unisinos, v. 8, p. $220-230$. 
Novaes, Antonio Galvão. (2007) Logística e Gerenciamento da Cadeia de Distribuição: Estratégia, Operação e Avaliação. $4^{\mathrm{a}}$ reimpressão. Rio de Janeiro: Elsevier.

Porter, Michael E. (1989) Vantagem Competitiva: criando e sustentando um desempenho superior. Rio de Janeiro: Campus.

Porter, M. E. (nov-dez/1998) Clusters and the new economics of competition. Harvard Business Review.

Porter, M. E. (april 12, 2001) Cluster and competitiveness: findings from the cluster mapping project. In: Corporate Strategies for the digital economy. Sloan Industry Centers. Cambridge.

Possas, Silvia. (1999) Concorrência e competitividade: notas sobre estratégia e dinâmica seletiva na economia capitalista. São Paulo: Hucitec.

Ricarte, Marcos António Chaves. (2002) A importância dos custos logísticos na cadeia de suprimentos. São Paulo: Widesoft Sistemas. Disponível em:

http://www.pauloangelim.com.br/artigos3 52.html. Acesso entre 20 a 30/12/2013.

Robles, Léo Tadeu. A prestação de serviços de logística integrada na indústria automobilística no Brasil: em busca de alianças logísticas estratégicas. Tese de Doutorado apresentada na USP. São Paulo, 2001.

Rozenfeld, Henrique; Forcellini, Fernando Antônio. Gestão de desenvolvimento de produtos: uma referência para a melhoria do processo. São Paulo: Saraiva, 2006. 542p.

SEBRAE apud Moraes, S. C. S. (2008) Análise da competitividade de um aglomerado produtivo pelo viés do empreendedorismo: uma proposta metodológica. Ponta Grossa. Disponível em http://www.pg.utfpr.edu.br/dirppg/ppgep/dissertac oes/arquivos/79/Dissertacao.pdf. Acesso em 2/8/2013.

Severino, Antônio Joaquim. (2007) Metodologia do trabalho científico. 23. ed. rev. e atual. São Paulo: Cortez.

Schmitz, H. (1994) Collective efficiency: growth path for small-scale industry. Brighton: IDS.

Scott, A. (1998) The geographic foundations of industrial performance. In A. Chandler, Jr., Hagstrom, P. and Solvell, O.,(eds.), The Dynamic Firm - The Role of Technology, Organization and Regions. Oxford: Oxford University Press, Chapter 16.

Stamer, Jörg Meyer. (2001) Estratégia de Desenvolvimento Local e Regional: Cluster, Política de Localização e Competitividade Sistêmica. Policy Paper.

Suzigan, W.; Furtado, J.; Garcia, R.; Sampaio, S. E. K. (out-dez/2003) Aglomerações industriais no Estado de São Paulo. Economia Aplicada, v. 5, n. 4, p. 698-717.

Suzigan, Wilson; Furtado, João; Garcia, Renato de Castro; Sampaio, Sérgio E. K. (jul/2004) Sistemas locais de produção: mapeamento, tipologia e sugestões de políticas. Revista de Economia Política, São Paulo, v. 24, n. 4, p. 543-562.

Yin, R. K. (2010) Estudo de Caso: planejamento e métodos $4^{\mathrm{a}}$ edição Porto Alegre Bookman.

Vasconcelos, Flávio C. de, Goldszmidt, Rafael G. B. e Ferreira, Fernando C. M. (ago/out/2005) Arranjos Produtivos. Economia, FGV-EAESP. Vol.4 nº3. Disponível em http://rae.fgv.br/sites/rae.fgv.br/files/artigos/3919.p df. Acessado entre 25/ a 28 de julho de 2014. 